\title{
Glioblastoma Occurred 20 Years after Cerebrospinal Irradiation: Case Report and Literature Review
}

\author{
S.H Touimi, I Mbarki, H Elkacemi, S Elmajjaoui, T Kebdani, N Benjaafar
}

\begin{abstract}
Radiation-induced gliomas represent a relatively rare but well-characterized entity in the neurooncologic literature. Age-related cerebral vulnerability could be a specific factor in the genesis of these complications.

The occurrence of a malignant glioma at the site of a preexisting medulloblastoma is exceptional.

Our purpose is to review the literature regarding the role of radiotherapy (RT) in the treatment of patients with radiation-induced malignant gliomas (RIMGs)

These findings support the notion that radiation-induced GBM should be considered a distinct clinical entity. Further understanding of multiple genetic changes after radiation will help protect patients who have already been cured of their original malignancy.
\end{abstract}

Index Terms - Glioblastoma, cerebrospinal, irradiation, medulloblastoma

\section{INTRODUCTION}

Radiotherapy is an important weapon in the treatment of cancers, but it can lead to rare dreadful late side effects such as radionecrosis or oncogenesis.

Radiation-induced glioma is a rare complication after cranial irradiation, described only in some cases and in small series. We report the case of probably radiation-induced glioblastoma in 23 years old patient treated at the age of 3 for medulloblastoma.

This is an atypical observation, due to latency time, tumor aggressiveness and rapid evolution.

\section{CASE REPORT}

A 23 years old patient treated at the age of 3 for medulloblastoma of posterior cranial fossa by surgery and adjuvant craniospinal radiation therapy.

The patient was followed regularly in consultation until the age of 10 and then was lost in sight.

13 years later, the patient presented an ataxia rapidly worsening associated to balance disturbances and pseudotumor cerebri. Cerebral MRI showed a right cerebellar infiltrating, compressive expansive process in favor of recurrence associated to left hemispheric cerebellar lesions compatible with secondary locations. A stereotactic biopsy with anatomopathological study and

TOUIMI Samia Hajar, Department Of Radiotherapy, National Institute Of Oncology, Rabat, Morocco

MBARKI Imane, Department Of Radiotherapy, National Institute Of Oncology, Rabat, Morocco

ELKACEMI Hanan, Department Of Radiotherapy, National Institute Of Oncology, Rabat, Morocco immunohistochemical complement showed a glioblastoma. The clinical examination found a patient altered in WHO 3 with left hemiparesis.

The evolution was marked by the appearance of respiratory distress with swallowing disorders. Drainage of hydrocephalus could not be achieved due to anesthetic difficulties. The patient had an emergency tracheotomy and started corticotherapy. Radiotherapy has not been proposed because of his general condition. The patient died 10 days later.
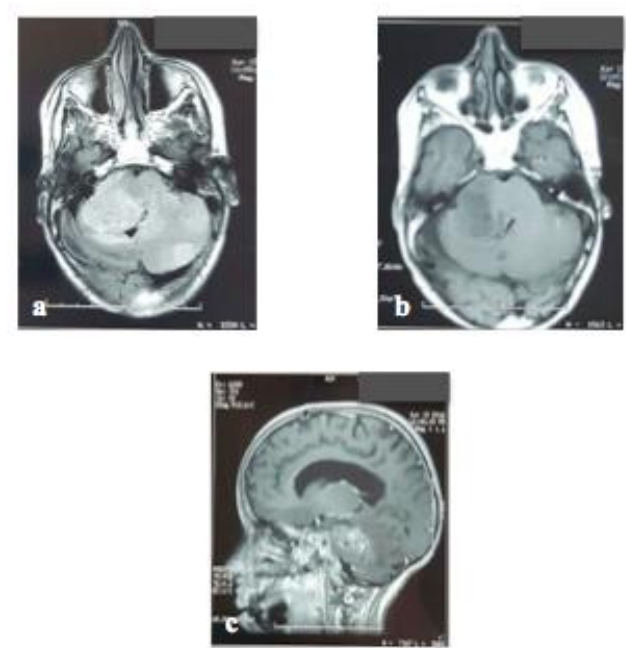

Figure: Cerebral MRI: a-b /axial plans c/ sagittal reconstruction

\section{DISCUSSION}

Glioblastomas (GBMs) are the second most common primary neoplasm of the central nervous system (CNS) in adults. Radiation-induced malignancies were defined in a classic study by Cahan et al. [1] four criteria for radiation-induced malignancies: 1-presence of tumor in a previously irradiated region, 2-sufficient latency time between the original and new tumors, generally measured in years, 3-histology of the new tumor must be distinct from the original, and 4-no history of disease predisposing to tumor development.

The Childhood Cancer Survivor Study consists of a cohort of 14361 five-year childhood cancer survivors [2]. An analysis of these patients showed that 40 went on to develop gliomas (including gliosarcomas, oligodendrogliomas, and juvenile pilocytic astrocytomas). Ninety percent of these 40 patients were originally treated for leukemia, CNS tumor, or lymphoma and $90 \%$ received some form of radiation.

In addition to treatment for malignant diseases, pediatric patients were also treated with ionizing radiation for benign conditions. A retrospective analysis of 10,834 pediatric 
patients treated with low-dose radiation (median estimated brain dose of $1.5 \mathrm{~Gy}$ ) for tinea capitis resulted in 21 cases of malignant tumors (defined as gliomas of all types, including ependymomas) [3].

A retrospective study of 4581 pediatric cancer patients in France treated with radiation indicates that many secondary malignancies actually occur in lower dose areas [4]. Specifically, $31 \%$ of secondary malignancies $(14 \%$ in the $\mathrm{CNS}$ ) occurred in volumes that received $<2.5 \mathrm{~Gy}$. Interestingly, in $6 \%$ of patients $(43 \%$ in the CNS) secondary malignancies occurred in volumes that received $<0.1 \mathrm{~Gy}$. However, more recent data using pediatric phantoms suggest that while doses may fall off rapidly near the target, peripheral doses remain relatively high with intensity modulated radiation therapy compared to $3 \mathrm{D}$ conformal radiation [5] - [6].

Some have suggested that the use of protons rather than $\mathrm{X}$-rays may reduce the risk of secondary malignancies [7].

A more recent study evaluated five RIG specimens using microarray analysis [8]

Three genes in particular were highly over-expressed, including ErB3 and Sox10 (both of which were previously implicated in astrocyte development) and PDGF $\alpha$. Inhibition of ErbB3 through disruption of post-translational processing increased radiosensitivity of glioma cells [9]. PDGFa conjugated with I-125 radioisotopes has been shown to preferentially accumulate in glioma cells [10]. Finally, Sox 10 was independently found to act synergistically with PDGFB to promote glioma development [11].

Radiation-induced gliomas do not necessarily represent a distinct clinical entity. The median survival of patients with RIGs is approximately 11 months with one-, 2-, and 5-year overall survival rates of $37 \%, 13 \%$, and $4 \%$, respectively [12].

A retrospective analysis by Paulino et al. [12] showed decidedly inferior outcomes in patients who did not receive re-irradiation for RIGs. Of 85 patients evaluated, 35 underwent re-irradiation with a mean dose of $50 \mathrm{~Gy}$ (range: $30-76 \mathrm{~Gy}$ ) with reported 2-year survival of $20.5 \%$. In contrast, the 50 patients who were not irradiated had a 2 -year survival of $3.0 \%$.

Classically, dose limits for partial brain and whole brain radiation have been set at 60 Gy and 50 Gy, respectively [13]. Mayer and Sminia found that re-irradiated normal brain tissue tolerated acumulative normalized total dose (NTDcumulative) of $>100$ Gy at conventional fractionation [14]. This was predicated on assumptions that normal brain was classified as a late-responding tissue $(\alpha / \beta=2 \mathrm{~Gy})$ and that fractionation was conventional ( 2 Gy per daily fraction).

When using active scanning protons, also known as intensity modulated proton therapy (IMPT), it is possible to reduce the dose to healthy tissue in pediatric patients [15] compared to comparable photon devices. [16]

A comprehensive search of the literature revealed 9 cases of gliomas 6 high [17] - [18] - [19] - [20] - [21] - [22] and 3 low
[23] - [24] - [25] grade) occurring 6 to 16 years following radiation for medulloblastomas.

Pearly and al reported a case of a supratentorial glioblastoma that occurred 13 years after radiation therapy for a cerebellar medulloblastoma. After a subtotal resection, he received radiation therapy consisting of 3000 rads to the whole brain, a booster dose of 1000 rads to the posterior fossa, and 2000 rads to the spine. The patient was again treated with X-irradiation: 3000 rads to the whole brain, a 1SOO-rad booster dose to the left parietal region, and 3000 rads to the spinal cord. He died 2years later. [26]

Schmidbauer and al reported development of a glioblastoma multiforme at the site of excision of a medulloblastoma 6 years earlier. The patient was operated on for a cerebellar medulloblastoma at the age of 13 years. Postoperative treatment included irradiation with a dose of 60 Gy from a 6-MeV x-ray source, accompanied and followed by nine courses of chemotherapy. Six years later, a glioblastoma multiforme was found at the original site of the medulloblastoma. The patient had a second surgery followed by radiotherapy to the posterior fossa (total dose $55 \mathrm{~Gy}$ ), supplemented by chemotherapy with a variety of cytotoxic drugs. [21]

Malde and al reported a rare case of a gliosarcoma occurring 8 years following treatment for a medulloblastoma. The patient was diagnosed with a medulloblastoma at the age of 13 years and received postoperative craniospinal radiotherapy with a dose of 35 Gy to the whole brain and spine along with an additional boost dose of $20 \mathrm{~Gy}$ to the posterior fossa. In view of the residual disease near the brain stem, she also received 12 monthly cycles of adjuvant chemotherapy. 8years later, she received three-dimensional conformal reirradiation (3DCRT) with a total dose of $30 \mathrm{~Gy}$ in six weekly fractions. [27]

Izci and al reported a case of multiform glioblastoma occuring 4years after medulloblastoma treatment. The 10-year-old girl had been treated by surgery and irradiation for a posterior fossa medulloblastoma. [28]

Yang and al reported a case occurring 10 years after treatment of cerebellar medulloblastoma. The patient was a 15-year-old boy who had undergone a gross-total removal of a medulloblastoma and received radiation therapy at the age of 5 years in total dose of 36 Gy of radiation to the brain, an additional 20 Gy to the primary tumor site as a focal boost, and 24 Gy to the spine followed by 10 courses of chemotherapy. He had experienced no tumor recurrences for 10 years until a new enhancing mass was found at the original site of the medulloblastoma.

Following its resection the new lesion was found to be a GBM and there was no evidence of a medulloblastoma. The second tumor developed at the same site as the previous one after a sufficient latent period and fulfilled the criteria for a radiation-induced neoplasm. [29]

\section{CONCLUSION}

The applied intensive multimodality treatment seems pivotal for development of the secondary tumor, either by 
influencing tumor remnants or primitive stem cells, or by inducing a malignant tumorde novo, resulting in a new tumor phenotype. As the number of long-term survivors of brain tumors increases due to intensive combined treatment, more examples of such a previously poorly appreciated tumor development might occur which require (very) long-term monitoring and surveillance.

\section{ACKNOWLEDGMENT}

We thank Radiotherapy department and our radiotherapist-s colleagues at national institute of oncology of Rabat who provided care and support for the patient.

\section{REFERENCES}

[1]. Cahan WG, Woodard HQ, Higinbotham NL, Stewart FW, Coley BL. Sarcoma arising in irradiated bone: report of 11 cases. 1948 Cancer. 1998; 82(1):6-34.

[2]. Neglia JP, Robison LL, Stovall M, et al. New primary neoplasms of the central nervous system in survivors of childhood cancer: a report from the childhood cancer survivor study. J Nat Can Inst.2006; 98(21):1528-1537.

[3]. Sadetzki S, Chetrit A, Freedman L, Stovall M, Modan B, Novikov I. Long-term follow-up for brain tumor development after childhood exposure to ionizing radiation for tinea capitis. Rad Res. 2005; 163:424-432.

[4]. Diallo I, Haddy N, Adjadj E, et al. Frequency distribution of second solid cancer locations in relation to the irradiated volume among 115 patients treated for childhood cancer. Int J Rad Biol Phys. 2009 ; 74(3):876-883.

[5]. Klein EE, Maserang B, Woord R, Mansur D. Peripheral doses from pediatric IMRT. Med Phys. 2006; 33(7):2525-2531.

[6]. Mansur DB, Klein EE, Maserang BP. Measured peripheral dose in pediatric radiation therapy: a comparison of intensity-modulated and conformal techniques. Radiother Oncol. 2007; 82:179- 184.

[7]. Hall EJ. Intensity-modulated radiation therapy, protons, and the risk of second cancers. Int J Radiat Biol Phys. 2006; 65(1):1-7.

[8]. Donson AM, Erwin NS, Kleinschmidt-DeMasters BK, Madden JR, Addo-Yobo SO, Foreman NK. Unique molecular characteristics of radiation-induced glioblastoma. J Neuropath Exp Neurol. 2007; 66(8): 740-749.

[9].Contessa JN, Bhojani MS, Freeze HH, Rehemtulla A, Lawrence TS. Inhibition of $\mathrm{N}$-linked glycosylation disrupts receptor tyrosine kinase signaling in tumor cells. Can Res. 2008; 68(10): 3803-3809.

[10]. Wester MD, Wasteson A, Lindstrom A. Targeting malignant glioma cells in vitro using platelet derived growth factor AA-based conjugates. J Drug Target. 2009; 7(4):268-277.

[11]. Ferletta M, Uhrbom L, Olofsson T, Ponten F, Westermark B Sox 10 has a broad expression pattern in gliomas and enhances platelet-derived growth factor-B-induced gliomagenesis. Mol Cancer Res. 2007; 5(9):891-897.

[12]. Paulino AC, Mai WY, Chintagumpala M, Taher A, Teh BS. Radiation-induced malignant gliomas: is there a role for reirradiation. Int J Rad Biol Phys. 2008; 71(5):1381-1387.

[13]. Emami B, Lyman J, Brown A, et al. Tolerance of normal tissue to therapeutic irradiation. Int J Radiat Oncol Biol Phys. 1991; 21(1):109-22.

[14]. Mayer R, Sminia P. Reirradiation tolerance of the human brain. Int J Radiat Oncol Biol Phys.2008; 70(5): 1350-1360.

[15]. Fogliata A, Yartsev S, Nicolini G, et al. On the performances of Intensity Modulated Protons, RapidArc and Helical Tomotherapy for selected paediatric cases. Radiat Oncol. 2009; 4:2.

[16]. Prasad G and Haas-Kogan D A. Radiation-induced gliomas. Expert Rev Neurother . 2009 October ; 9(10)

[17]. Furuta T, Sugui K, Tamiya $T$, Matsumoto $\mathrm{K}$, Ohmoto $\mathrm{T}$. Malignant cerebellar astrocytoma developing 15 years after radiation therapy for a medulloblastoma. Clin Neurol Neurosurg 1998 ; 100:56-59

[18]. Klergia E, Sher JH, Nallainathan SK, Stein SC, Sacher M. Development of cerebellar malignant astrocytoma at site of a medulloblastoma treated 11 years earlier. J Neurosurg 1978; 49:445-449

[19]. Pearl GS, Mirra SS, Miles ML. Glioblastoma multiforme occurring 13 years after treatment of a medulloblastoma. Neurosurgery $1980 ; 6: 546-551$

[20]. Safneck JR, Napier LB, Halliday WC. Malignant astrocytoma of the optic nerve in a child. Can J Neurol Sci $1993 ; 19: 498-503$

[21]. Schmidbauer M, Budka H, Bruckner R, Vorkapic P. Glioblastoma developing at the site of a cerebellar medulloblastoma treated 6 years earlier.J Neurosurg $1987 ; 67: 915-918$

[22]. Van Calenbergh F, D’Haen B, Dom R, Menten J, Plets C. Secondary supratentorial anaplastic astrocytoma following treatment of medulloblastoma. Eur J Paediatr Neurol 1999; $3: 177-180$

[23]. Cohen MS, Kushner MJ, Dell S. Frontal lobe astrocytoma following radiotherapy for medulloblastoma. Neurology 1981 ; 31:616-619

[24]. Anderson JR, Treip CS. Radiation induced intracranial neoplasms. Cancer1984; 53:426-429

[25]. Osmui AK, McLendon RE, Tien RD, Friedman HS, Graham M Hockenberger B, Halperin EC, Oakes WJ. Well-differentiated astrocytoma occurring 9 years after radiation therapy for medulloblastoma. Clin Neuropathol1994 ; 13:281-285

[26]. Pearl G S, Mirra S S, Miles M M. Glioblastoma Multiforme Occurring 13 Years after Treatment of a Medulloblastoma. Neurosurgery, 1980 ; 6: 546-551

[27]. Malde R, Jalali R, Muzumdar D, Shet T, Kurkure P. Gliosarcoma occurring 8 years after treatment for a medulloblastoma. Childs Nerv Syst (2004) 20:243-246

[28]. Izci Y, Akay M K, Gurkanlar D, Deveci M S. Radiation-induced glioblastoma multiforme following surgery for medulloblastoma in a child with neurofibromatosis-1:Case report. Turkish Neurosurgery, 2005, Vol: 15 No: 1, 36-39

[29]. Yang S-Y, Wang K-C, Cho B-K, Kim Y-Y, Lim S-Y, Park S-Y, Kim I H, Kim S-K. Radiation-induced cerebellar glioblastoma at the site of a treated medulloblastoma. J Neurosurg (Pediatrics 4) $2005 ; 102: 417-422$ 